\title{
Nanolocalized UV source of laser radiation
}

\author{
P.N. Melentiev ${ }^{1, *}$, A.E. Afanasiev ${ }^{1}$, A.A. Kuzin ${ }^{1,2}$, and V.I. Balykin ${ }^{1}$ \\ ${ }^{1}$ Institute for Spectroscopy RAS, 108840 Moscow, Troitsk, Russia \\ ${ }^{2}$ Moscow Institute of Physics and Technology, 141700 Dolgoprudny, Russia
}

\begin{abstract}
We show the results of optical nonlinear interaction of laser light with plasmonic nanostructures. Creation of a nanolocalized source of UV radiation $(\lambda=260 \mathrm{~nm})$ of the record high intensity was demonstated. Possibility of use of the source for biomedical applications was shown.
\end{abstract}

Basic elements of nanoplasmonics are formed by metal structures having nano meter-scale geometry. Interaction of nanostructures with laser light at the frequencies of the localized plasmonic resonances leads to a high values of electric field near the nanostructure more than the amplitude of the incident light wave. Thus, nanostructure helps to localize light energy on a size considerably smaller compared to the wavelength [1]. There are different practical applications of the local field enhancement known such as optical microscopy with nano-meter resolution, biosensors, optical nanolithography, photovoltaics, light registration and photodetectors.

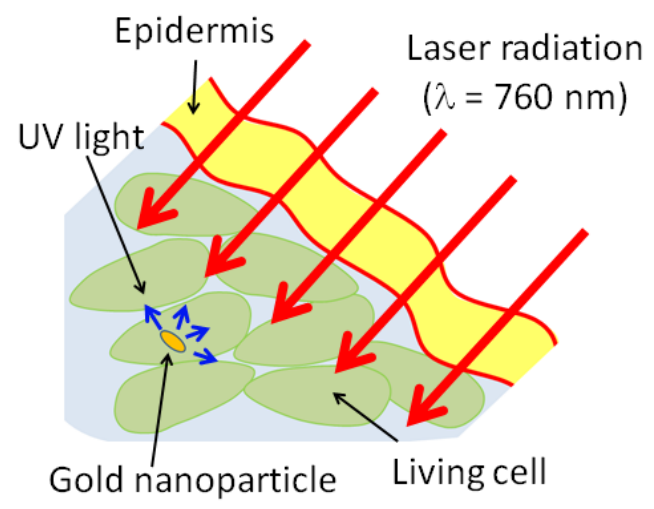

Fig. 1. Schematic figure of the application of the strong optical nonlinearity of plasmonic nanostructures for the UV treatment of living cell: UV radiation is formed by plasmonic nanostructure as a result of the harmonics generation. Radiation at fundamental frequency has the wavelength corresponding to a maximum value of the transmittance spectral window of a biological tissue.

\footnotetext{
* Corresponding author: melentiev@isan.troitsk.ru
} 
A separate practical interest is a generation of harmonics by nanostructures in the UV spectral range. First of all this helps to realize nanolocalized sources of UV radiation, needed for nano-diagnostics and nanolithography. As a second, high optical nonlinearity of nanostructures in the UV spectral range can be used in the photo induced therapy of living cells (Fig. 1). Generation of a radiation in the UV spectral range by plasmonics nanostructures are not studied well. There are currently only several experimental papers where was demonstrated generation of third harmonics in the UV spectral range.

In this talk we present our results of optical nonlinear interaction of a femtosecond laser radiation having duration of $6 \mathrm{fs}$ and $60 \mathrm{fs}$ with individual plasmonic nanostructures made in aluminum and gold nanofilms. Chosen geometry of nanostructures helps to realize plasmonic resonance at the frequency of laser radiation, that leads to generation of the third harmonics in the deep UV spectral range, with a wavelength equals to $260 \mathrm{~nm}$. We show that nanostructures made in gold nanofilm can be used for building the nanolocalized sources of UV radiation with a record high intensity.

This work was supported by the Russian Science Foundation (project No.14-12-00729).

\section{References}

1. L. Novotny, N. van Hulst, Nature Photonics 5, 83 (2011)

2. P. Sasanpour, B. Rashidian, B. Rashidian, M. Vossoughi, Nano 5, 325 (2010) 